Raimundo Antonio da Silva ${ }^{1}$

Antonio Carlos Ribeiro ${ }^{2}$

\title{
Associação entre Espondiloartrose lombar e trabalho pesado
}

\author{
Association between lumbar spondiloarthrosis and heavy \\ physical work
}

1 Professor Associado do Departamento de Saúde Pública da Universidade Federal do Maranhão, UFMA.

${ }^{2}$ Médico do Trabalho e Ortopedista do Hospital Tarquínio Lopes Filho - HTLF, São Luís - MA

\section{Contato:}

Dr. Antonio Carlos Ribeiro

Rua Augusto dos Anjos, quadra J, no 26

Bairro: Ipase. São Luís - Maranhão

CEP: 65061-090

E-mail:

antonium@hotmail.com

\section{Resumo}

Objetivo: Estudar uma possível associação entre espondiloartrose lombar e trabalho pesado. Metodologia: Estudo epidemiológico transversal realizado no período de outubro/04 a outubro/05, baseado em casos atendidos por um hospital público de São Luís, MA, Brasil. Trabalhadores (1256) encaminhados ao serviço de ortopedia foram submetidos à História Ocupacional, Exame Físico Ortopédico e Radiografia de Coluna Lombar e Lombossacra em AP/P e Oblíqüa. Critérios de inclusão e exclusão selecionaram 456 trabalhadores de ambos os gêneros, na faixa etária de 28 a 48 anos. Estes foram agrupados, conforme sua ocupação, em trabalho pesado e não pesado e submetidos aos critérios diagnósticos de espondiloartrose lombar. Resultados: A associação entre trabalho pesado e espondiloartrose lombar foi realizada através do cálculo da razão de prevalências, cujo resultado mostrou-se elevado, evidenciando uma possível associação. A interferência de outras variáveis como possível viés de confundimento, tais como idade, gênero e peso corporal (IMC), foram controladas na fase de análise de dados através do teste de Mantel-Haenzel. O resultado final mostrou que não houve interferência dessas variáveis. Conclusões: Este estudo indicou uma possível associação entre exposição a trabalho pesado e espondiloartrose lombar.

Palavras-chave: espondiloartrose lombar, trabalho pesado, trabalho não pesado.

\begin{abstract}
Objective: To study a possible association between lumbar spondiloarthrosis and heavy physical work. Method: Epidemiological transversal study was performed from October/04 to October/05 based on some cases cared for at a public hospital in São Luís, Maranhão, Brazil. Workers (1256) sent to the hospital orthopaedic service were submitted to occupational history, physical orthopedic exam and vertebral lumbar and lumbossacral radiographs in $A P / P$ and oblique view. A total of 456 male and female workers between 28 and 48 years of age were selected according to evaluation inclusion / exclusion criteria. They were categorized according to their heavy and non-heavy work and were submitted to the diagnostic criteria of lumbar spondiloartrhosis. Results: The association between heavy work and lumbar spondiloarthrosis was calculated using the prevalence ratio, which was high, evidencing a possible association. The interference of other variables as possible confounding factors, such as sex, age and body weight (BMI) were controlled in the data analysed using the Mantel-Haenzel test. The final result showed no interference of the mentioned variables. Conclusions: this study showed a possible association between heavy work exposure and lumbar spondiloarthrosis.
\end{abstract}

Keywords: lumbar spondiloarthrosis, heavy physical work, non heavy physical work.
Recebido: 03/10/2008

Revisado: 02/03/2009

Aprovado: 02/03/2009 


\section{Introdução}

A relação entre doença e ambiente de trabalho foi bem estabelecida a partir de 1700 , quando o médico Bernadino Ramazzini publicou em Módena, na Itália, o seu livro De Morbis Artificum Diatriba, considerado um clássico da literatura médica, no qual relaciona doenças que ocorrem em mais de cinqüenta ocupações (SKROBONJA; KONTOSIC, 2002). Ramazzini deu um novo enfoque a esta questão quando sugeriu que os médicos acrescentassem na anamnese do paciente a pergunta: Qual a sua ocupação? (MENDES, 1995).

O trabalho humano possui três aspectos: físico, cognitivo e psíquico, cada um deles, individualmente ou inter-relacionados podem determinar uma sobrecarga (WISNER, 1994).

Elementos do processo de trabalho, denominados por Laurell e Noriega (1989) como cargas de trabalho, podem interagir com o corpo do trabalhador gerando adaptações que se traduzem em desgastes e perda da capacidade corporal e psíquica.

Romerth e Rutenfranz (apud SELL, 1994, p. 270272), classificam o trabalho em duas formas básicas: 1 - trabalho predominantemente corporal, muscular e senso motor e 2 - trabalho não predominantemente corporal. No primeiro tipo, a atividade seria caracterizada pela geração de forças e solicitação sensomotora e muscular. O segundo tipo seria caracterizado pela solicitação dos órgãos dos sentidos, das capacidades e habilidades mentais.

Khalil et al. (1993), descrevendo lombalgia crônica relacionada com as características do trabalho, classificam as profissões pela exigência de trabalho pesado e de trabalho moderado e sedentário. Citam como eventos relacionados aos distúrbios da coluna lombar os atos de levantar, carregar, puxar e empurrar pesos. Thayyari e Smith (1997), em estudo sobre a organização e o processo de trabalho, evidenciam que fatores ergonômicos como força, postura e vibração podem determinar lombalgia, osteoartrite e tenossinovites.

A espondiloartrose lombar é um tipo de doença degenerativa da coluna vertebral que inclui duas patologias distintas, porém interelacionadas: a espondilose ou doença degenerativa discal e a osteoartrite das articulações interapofisárias posteriores (LEMOS; RISO, 1981; SALTER, 1985; NUNES, 1989). O processo degenerativo discal pode ter início a partir da segunda década de vida e a sua etiopatogênese está relacionada a alterações que ocorrem no núcleo pulposo (LEBKOWISKI, 2002). Fissuras ocorrem no anel fibroso com posterior diminuição do espaço intervertebral e formação de osteófitos (LEMOS; RISO, 1981; NUNES, 1989).

As articulações interapofisárias posteriores podem sofrer do mesmo processo degenerativo articular ou osteoartrose de uma articulação sinovial. A cartilagem articular das facetas articulares sofre fibrilação, amo- lecimento e o osso subcondral eburnifica-se podendo aparecer osteófitos (McCARTHY; FRASSICA, 1998).

Lebkowisk (2002) enfatiza que a idade é o principal fator etiológico da espondiloartrose lombar. Knoplich (1995) afirma que as alterações radiográficas, como diminuição de espaço intervertebral e osteofitose em pacientes após a quarta década de vida, estão mais relacionadas ao envelhecimento.

Battie, Viedman e Parent (2004) afirmam que trabalho pesado tem pouca influência na determinação de doença degenerativa discal e selecionam fator genético como principal agente etiológico dessa patologia. Viedman, Nurmien e Trap (1990) encontraram associação entre espondiloartrose lombar e trabalhadores que exerceram trabalho sedentário. Entretanto, Fazzi e Barros Filho (1992) mostram que há evidências clínicas de que solicitações de sobrecarga e repetitividade atuando na região lombar determinam, inicialmente, alterações musculares, ligamentares, capsulares e, finalmente, alterações degenerativas da coluna vertebral, como espondiloartrose.

Do mesmo modo, Seidler et al. (2001) sugerem que exposição ocupacional cumulativa em atividades como levantamento e carregamento de peso aumentam o risco de desenvolvimento de espondiloar trose lombar.

A relação entre forças mecânicas e alterações no disco intervertebral já foi pesquisada em laboratórios de biomecânica. O trabalho clássico de Nachemson e Elfstrom (1970) verificou que a pressão intradiscal é variável de acordo com a postura. Matsumoto et al. (1999) verificaram que forças mecânicas de stress atuando sobre o núcleo pulposo e o annulus fibroso promovem uma alteração celular no disco intervertebral. Lotz et al. (1998), por sua vez, demonstraram que forças de compressão atuando no disco intervertebral promovem uma desorganização na estrutura do annulus fibroso, aumento na apoptose celular e diminuição da regulação do colágeno tipo II.

Ribeiro (1979), em pesquisa sobre doenças ocupacionais e acidente de trabalho em varas judiciais de Brasília, encontrou 23,4\% de patologias de coluna vertebral devido, principalmente, a espondiloartrose e dentre os trabalhadores mais acometidos estavam os que exerciam trabalho pesado.

O Ministério da Saúde, através do Manual de Procedimentos para os Serviços de Saúde, orienta que pode haver um nexo de causalidade entre artrose e trabalho. Apesar da patologia artrósica possuir uma multicausalidade, "o trabalho pode ser considerado como concausa” (BRASIL, 2001, p. 446-447)

Esta pesquisa é uma contribuição para a economia da saúde do trabalhador e suas implicações sociais e previdenciárias, uma vez que teve por objetivo evidenciar uma possível associação entre trabalho fisicamente pesado e espondiloartrose lombar. Estudos que demonstram associação entre fatores de risco 
presentes em algumas profissões e doenças da coluna vertebral são de grande importância para os trabalhadores que exercem trabalho pesado, pois o nexo de causalidade é baseado em evidências epidemiológicas.

\section{Metodologia}

O delineamento utilizado foi do tipo transversal. Trabalhadores portadores de lombalgia crônica, em um total de 1256, encaminhados ao ambulatório de Ortopedia do Hospital Tarquínio Lopes Filho Hospital Geral de São Luís - Maranhão, no período de outubro de 2004 a outubro de 2005, após assinatura de termo de consentimento livre e esclarecido, foram submetidos aos seguintes procedimentos: ficha de identificação, história clínico-ocupacional, exame físico ortopédico e radiografia de coluna lombar e lombossacra em AP/P e Oblíqüas.

O estudo teve como critérios de inclusão: trabalhadores de ambos os gêneros na faixa etária de 28 a 48 anos; história de 10 anos ou mais na mesma profissão ou ocupação. Foram excluídos do estudo trabalhadores com história de causas secundárias de espondiloartrose lombar tais como: espondilopatias inflamatórias, neoplasias, infecção óssea vertebral, fratura-luxação de coluna vertebral lombar, doenças osteometabólicas, doenças hematológicas e escoliose lombar. Dos 1256 trabalhadores avaliados, 456 atenderam aos critérios de inclusão definidos acima.

A escolha da faixa etária de 28 a 48 anos deve-se ao fato de que a atividade ocupacional inicia-se aos 18 anos e, somando-se dez anos de atividade laboral na mesma ocupação teríamos um tempo de exposição previsto como possível e suficiente para determinar alterações degenerativas esqueléticas, segundo a experiência dos autores. Por outro lado, a espondiloartrose é uma doença cuja incidência e prevalência aumenta com a idade, sendo bastante comum em pessoas com 60 anos ou mais (McCARTHY; FRASSICA, 1998). A escolha de 10 anos ou mais na mesma profissão reside no fato de que indivíduos que trocam de profissões deixam de sofrerem exposição aos mesmos fatores de risco podendo confundir os resultados.

As variáveis independentes estudadas foram as seguintes: tipo de trabalho, gênero, idade e peso corporal. Quanto ao tipo de trabalho, os participantes foram categorizados em: trabalho pesado e trabalho não pesado. O tipo de trabalho foi definido levando-se em consideração a categoria ocupacional do trabalhador. As profissões com trabalho predominantemente corporal foram incluídas como trabalho pesado e as profissões com trabalho não predominantemente corporal foram incluídas como trabalho não pesado. Quanto à idade, os trabalhadores foram distribuídos nas seguintes categorias: 28 a 39 anos e 40 a 48 anos. Até 40 anos a prevalência de doença degenerativa articular, incluindo espondiloartrose lombar varia de $10 \%$ a $20 \%$ na popu- lação geral, aumentando progressivamente após esta faixa etária (McCARTHY; FRASSICA, 1998). Quanto ao peso corporal, os trabalhadores foram classificados de acordo com o índice de massa corpórea (IMC) em: IMC $<25 \mathrm{~kg} / \mathrm{m}^{2}$ e IMC $\geq 25 \mathrm{~kg} / \mathrm{m}^{2}$, utilizando-se os critérios recomendados pela OMS (WORD HEALTH ORGANIZATION, 1995).

A variável dependente foi espondiloartrose lombar. Foram considerados portadores de espondiloartrose lombar, os trabalhadores que apresentaram: doença degenerativa discal e/ ou osteoartrose de articulações interfacetárias posteriores da coluna lombar. A presença ou ausência de espondiloartrose lombar foi confirmada através de radiografia simples da coluna lombar e lombossacra em AP/P e oblíqüas, seguindo os seguintes critérios para espondiloartrose lombar: presença de osteofitose na coluna lombar com ou sem diminuição do espaço intervertebral e/ou osteoartrose interfacetária posterior caracterizada por diminuição de espaço articular interapofisário, irregularidade das facetas articulares e presença de osteófitos. A confirmação diagnóstica foi realizada por dois médicos ortopedistas e o laudo radiológico de presença ou ausência de espondiloartrose.

A escolha do método diagnóstico baseou-se no fato de todos os pacientes apresentarem lombalgia crônica e por ser a radiografia simples da coluna lombar um exame de baixo custo, rápido, de fácil interpretação e de grande especificidade para espondiloartrose lombar.

A razão de prevalências entre tipo de trabalho e espondiloartrose lombar foi calculada com a finalidade de verificar a possibilidade de exposição a trabalho pesado ser fator de risco para esse agravo. $\mathrm{O}$ teste não paramétrico do Qui-Quadrado também foi utilizado. Para avaliação do viés de confundimento entre as variáveis espondiloartrose, trabalho pesado, peso corporal e idade, utilizou-se estratificação através do teste de Mantel-Haenszel. O programa Bio-Estat 2,0 foi utilizado na análise estatística (AYRES et al., 2000).

O estudo foi realizado em conformidade com a resolução 196/96 do Ministério da Saúde e foi aprovado pelo comitê de ética da Universidade Federal do Maranhão. Após devidamente explicado, um termo de consentimento livre e esclarecido foi assinado pelos participantes.

\section{Resultados}

Do total de 456 trabalhadores, $239(52,4 \%)$ eram do gênero feminino e $217(47,6 \%)$ do gênero masculino. Trabalho pesado era exercido por $213(46,7 \%)$ trabalhadores, enquanto $243(53,3 \%)$ exerciam trabalho não pesado. Em relação à idade, 266 (58,3\%) trabalhadores estavam na faixa etária entre 28 e 39 anos e 190 (41,7\%) na faixa etária entre 40 e 48 anos; a 
média de idade dos trabalhadores da amostra foi de quarenta e dois anos. Quanto ao peso corporal, 224 $(49,2 \%)$ indivíduos possuíam IMC $<25 \mathrm{~kg} / \mathrm{m}^{2}$ e 232 $(50,8 \%)$ apresentaram IMC $\geq 25 \mathrm{~kg} / \mathrm{m}^{2}$. A Tabela 1 evidencia os tipos de ocupações dos trabalhadores e sua classificação em trabalho pesado e trabalho não pesado.

A Tabela 2 apresenta a razão de prevalências, que indica uma possível associação entre trabalho pesado e espondiloartrose lombar.

A Tabela 3 mostra maior prevalência de espondiloartrose lombar nos trabalhadores que exerciam trabalho pesado, tanto na faixa etária de 28 a 39 anos como na faixa etária de 40 a 48 anos. Quando aplicado o teste de Mantel Haenzel, o resultado mostrou-se altamente significativo ( $\mathrm{p}<0,0001)$, para um nível de significância de 0,05 , o que indica que a faixa etária não influenciou o resultado da possível associação entre trabalho pesado e espondiloartrose lombar.
A Tabela 4 evidencia que não houve diferença significativa na prevalência de espondiloartrose lombar em relação ao gênero, porém tanto em homens quanto em mulheres a espondiloartrose lombar teve maior prevalência nos que exerciam trabalho pesado. Quando aplicado o teste de Mantel Haenzel, o resultado mostrou-se muito significativo ( $p<0,0001)$, o que indica que a variável gênero não influenciou o resultado da possível associação entre trabalho pesado e espondiloartrose lombar.

A Tabela 5 mostra que a prevalência de espondiloartrose lombar foi mais elevada em trabalhadores com $\mathrm{IMC} \geq 25 \mathrm{~kg} / \mathrm{m}^{2}$ de modo significativo, no entanto, quando relacionada ao tipo de trabalho, a espondiloartrose lombar tanto em trabalhadores com IMC $<25 \mathrm{~kg} / \mathrm{m}^{2}$ como naqueles com IMC $\geq 25 \mathrm{~kg} / \mathrm{m}^{2}$ teve maior prevalência naqueles que exerciam trabalho pesado, o que indica que não houve influência do peso corporal (IMC) no resultado de uma possível associação entre espondiloartrose lombar e trabalho pesado, quando aplicado o teste de Mantel-Haenzel.

Tabela 1 Distribuição de trabalhadores portadores de lombalgia crônica, de acordo com suas ocupações e classificação em trabalho pesado e não pesado, São Luís, MA, 2004

\begin{tabular}{lclc}
\hline \multicolumn{1}{c}{ Trabalho pesado } & $n$ & Trabalho não pesado & $n$ \\
\hline Estivador & 20 & Auxiliar de escritório & 75 \\
Servente de pedreiro & 30 & Agente administrativo & 110 \\
Pedreiro & 30 & Professor & 46 \\
Lavrador & 83 & Bancário & 12 \\
Pescador & 20 & & \\
Gari & 30 & & 243 \\
\hline Total & 213 & Total & \\
\hline
\end{tabular}

Tabela 2 Trabalho pesado como possível fator de risco para espondiloartrose lombar, São Luís, MA, 2004

\begin{tabular}{lcccccc}
\hline \multirow{2}{*}{ Tipo de trabalho } & Espondiloartrose lombar & Total & Taxa de prevalência (\%) & Razãode Prevalências (a/b) & Intervalo de confiança \\
& Presença & Ausência & & & & \\
\hline Pesado & 169 & 44 & 213 & 79,3 (a) & 6,66 & $4,96,41$ \\
Não pesado & 29 & 214 & 243 & 11,9 (b) & \\
\hline Total & 198 & 258 & 456 & 43,4 & \\
\hline
\end{tabular}


Tabela 3 Prevalência de espondiloartrose lombar (EL) em possível associação com trabalho pesado nas faixas etárias de 28 a 39 anos e 40 a 48 anos, São Luís, MA, 2004

\begin{tabular}{|c|c|c|c|c|c|c|c|}
\hline \multirow{4}{*}{ Tipo de trabalho } & \multicolumn{4}{|c|}{ Faixa etária } & \multirow{4}{*}{ Total } & \multirow{2}{*}{\multicolumn{2}{|c|}{$\begin{array}{l}\text { Taxa de prevalência } \\
\text { (\%) }\end{array}$}} \\
\hline & & & & & & & \\
\hline & \multicolumn{2}{|c|}{28 a 39 anos } & \multicolumn{2}{|c|}{40 a 48 anos } & & \multirow[t]{2}{*}{28 a 39 anos } & \multirow[t]{2}{*}{40 a 48 anos } \\
\hline & $c / E L$ & $s / E L$ & $c / E L$ & $s / E L$ & & & \\
\hline Pesado & 83 & 29 & 89 & 12 & 213 & 74,1 & 88,1 \\
\hline Não pesado & 2 & 152 & 24 & 65 & 243 & 1,29 & 26,9 \\
\hline Total & 85 & 181 & 113 & 77 & 456 & 31,9 & 59,4 \\
\hline
\end{tabular}

Tabela 4 Prevalência de espondiloartrose lombar (EL) em possível associação com trabalho pesado nos gêneros masculino e feminino, São Luís, MA, 2004

\begin{tabular}{lccccccc}
\hline \multicolumn{1}{c}{ Tipo de trabalho } & \multicolumn{3}{c}{ Gênero } & \multicolumn{3}{c}{ Taxade prevalência } \\
& feminino & masculino & Total & feminino & masculino \\
& c/EL & s/EL & c/EL & S/EL & & & \\
\hline Pesado & 60 & 20 & 104 & 29 & 213 & 75,0 & 78,1 \\
Não pesado & 17 & 142 & 17 & 67 & 243 & 10,6 & 20,3 \\
\hline Total & 77 & 162 & 121 & 96 & 456 & 32,2 & 55,7 \\
\hline
\end{tabular}

Tabela 5 Prevalência de espondiloartrose lombar (EL), em possível associação com trabalho pesado em trabalhadores com IMC $<25 \mathrm{~kg} / \mathrm{m} 2$ e IMC $\geq 25 \mathrm{~kg} / \mathrm{m} 2$, São Luís, MA, 2004

\begin{tabular}{|c|c|c|c|c|c|c|c|}
\hline \multirow{4}{*}{ Tipo de trabalho } & \multicolumn{4}{|c|}{$I M C$} & \multirow{4}{*}{ Total } & \multirow{2}{*}{\multicolumn{2}{|c|}{$\begin{array}{l}\text { Taxa de prevalência } \\
\text { (\%) }\end{array}$}} \\
\hline & & & & & & & \\
\hline & \multicolumn{2}{|c|}{$<25 \mathrm{~kg} / \mathrm{m}^{2}$} & \multicolumn{2}{|c|}{$\geq 25 \mathrm{~kg} / \mathrm{m}^{2}$} & & $<25 \mathrm{~kg} / \mathrm{m}^{2}$ & $\geq 25 \mathrm{~kg} / \mathrm{m}^{2}$ \\
\hline & $c / E L$ & $s / E L$ & $c / E L$ & $s / E L$ & & & \\
\hline Pesado & 71 & 31 & 91 & 20 & 213 & 69,6 & 81,9 \\
\hline Não pesado & 16 & 106 & 20 & 101 & 243 & 13,1 & 16,5 \\
\hline Total & 87 & 137 & 111 & 121 & 456 & 38,8 & 47,8 \\
\hline
\end{tabular}

\section{Discussão}

O presente estudo evidenciou que exposição a trabalho pesado pode ser um possível fator de risco para espondiloartrose lombar. A razão de prevalências foi elevada, demonstrando que a prevalência de espondiloartrose em trabalhadores que exercem atividades ocupacionais caracterizadas como trabalho pesado é maior que a prevalência em trabalhadores que não exercem trabalho pesado.
Em nosso meio, Mazzoni e Couto (1987) observaram que sinais radiológicos de discopatia degenerativa e osteoartrose vertebral foram mais evidentes em trabalhadores de serviço pesado quando comparados com trabalhadores de serviço sedentário.

Fernandes e Carvalho (2000) relataram resultados semelhantes em um estudo transversal em que a prevalência de patologia de disco intervertebral foi maior em trabalhadores com manuseio habitual de cargas em relação aos trabalhadores com manuseio ocasional de cargas. 
Do mesmo modo, Elsner, Niehaus e Beck (1997) demonstraram que manuseio de cargas pesadas determinam risco relativo elevado para espondiloartrose em ambos os gêneros. Igualmente, Seidler et al. (2001) encontraram maior risco de ser desenvolvida doença degenerativa lombar em trabalhadores com sobrecarga repetitiva sobre a coluna lombar.

Viedman, Nurmien e Trap (1990), em estudo realizado com cadáveres, encontraram uma maior associação de discopatia degenerativa com trabalho sedentário, e Batié, Viedman e Parent (2004) demonstraram que fatores genéticos possuem maior influência na determinação de patologia discal do que fatores ocupacionais.

Quando consideramos variáveis como a idade, verificamos que os resultados deste estudo evidenciaram que a espondiloartrose teve prevalência elevada em trabalhadores que exerciam trabalho pesado tanto na faixa etária de 28 a 39 anos como na faixa etária de 40 a 48 anos, o que indica que não há influências dessas faixas etárias na possível associação entre trabalho pesado e espondiloartrose lombar, o que foi confirmado pelo teste de Mantel-Haenzel.

Lebkowisk (2002) enfatiza que idade elevada é o principal fator etiológico da degeneração discal lombar. Entretanto, McCarthy e Frassica (1998) consideram que as doenças degenerativas articulares, de um modo geral, não estão necessariamente vinculadas ao envelhecimento e múltiplos fatores, inclusive ocupacionais, estão envolvidos em sua etiopatogênese. Pincus (2001) e Birchfield (2001) afirmam o mesmo com relação à espondiloartrose.

Em relação ao gênero, os resultados mostraram que a prevalência de espondiloartrose lombar não apresentou diferença estatística, contrariando os resultados de Simmons et al. (1996) que encontraram maior freqüência de discopatia degenerativa entre mulheres. Quando comparada, a prevalência de espondiloartrose e tipo de trabalho em ambos os gêneros, verificou-se que tanto no masculino como no feminino houve maior prevalência de espondiloartrose lombar em trabalhadores que exerciam trabalho pesado, evidenciando que a distribuição por gênero não influenciou o resultado da

\section{Referências}

AYRES, M. et al. Bio-Estat 2.0 - Aplicações estatísticas nas áreas das ciências biológicas e médicas. Belém: MCT - CNPq, 2000.

BATTIÉ, M. C.; VIEDMAN, T.; PARENT, E. Lumbar disc degeneration: epidemiology and genetic influences. Spine, United States, v. 29, n. 23, p. 26792690, Dec. 2004.

BIRCHFIELD, P. C. Ostheoarthritis overview. Geriatric Nursing, v. 22, n. 3, p. 124-130, May-June 2001. possibilidade de trabalho pesado ser fator de risco para espondiloartrose lombar.

Quanto ao peso corporal, o resultado evidenciou que houve uma prevalência significantemente maior de espondiloartrose lombar em trabalhadores com excesso de peso (IMC $\geq 25 \mathrm{~kg} / \mathrm{m}^{2}$ ), confirmando o estudo de Liuke et al. (2005) que afirmam que o excesso de peso aumenta risco de patologia degenerativa do disco intervertebral lombar. No entanto, quando verificada a influência da variável peso corporal na possível associação entre trabalho pesado e espondiloartrose lombar, observou-se que, para trabalhadores que exerciam trabalho pesado, a espondiloartrose lombar teve maior prevalência, tanto naqueles com IMC $<25 \mathrm{~kg} / \mathrm{m}^{2}$ como naqueles com IMC $\geq 25 \mathrm{~kg} / \mathrm{m}^{2}$, o que mostra que não ocorreu influência do peso corporal na possível associação entre trabalho pesado e espondiloartrose lombar.

Nesta pesquisa, verificamos uma possível associação significativa entre exposição a trabalho pesado e espondiloartrose lombar. Entretanto, este estudo foi um delineamento transversal e, apesar do viés de confundimento com idade, gênero e peso corporal ter sido controlado na fase de análise de dados, os resultados, pela natureza do desenho do estudo, não definem uma associação de causalidade. Estudos longitudinais para estabelecer a relação entre fator de risco e doença vertebral lombar, sobretudo relacionando o tempo de exposição e o aparecimento dos primeiros sinais e sintomas da espondiloartrose lombar, são necessários.

Evidências de que fatores decorrentes da organização e do processo de trabalho determinam discopatia degenerativa lombar e osteoartrose interfacetária lombar em algumas categorias profissionais que exercem trabalho pesado é de grande importância para o campo da saúde do trabalhador, principalmente em seus aspectos legais e previdenciários, sobretudo quando considera-se que, em sua grande maioria, os trabalhadores que exercem trabalho pesado não possuem outra opção ocupacional, tornando-se incapacitados precocemente para suas atividades laborais, acarretando altos custos sociais e previdenciários.

BRASIL. Ministério da Saúde. Organização PanAmericana de Saúde. Doenças relacionadas ao trabalho: manual de procedimentos para os serviços de saúde. Brasília: Ministério da Saúde, 2001.

ELSNER, G.; NIENHAUS, A.; BECK, W. Occupationally - induce degenerative discopathies in the area of the lumbar spine. Sozocial - und Praventivmedizin, Switzerland, v. 42, n. 3, p. 144-154, 1997.

FAZZI, A.; BARROS FILHO, T. E. P. Lombalgia do compartimento posterior: importância no processo 
degenerativo. Revista Brasileira de Ortopedia. São Paulo, v. 27, n. 3, p. 106-112, mar. 1992.

FERNANDES, R. C. P.; CARVALHO, F. M. Doença do disco intervertebral em trabalhadores de perfuração de petróleo. Cadernos de Saúde Pública, Rio de Janeiro, v. 16, n. 3, p. 661-669, set. 2000.

KHALIL, T. M. et al. Ergonomics in back pain: a guide to prevention and rehabilitation. New York: Van Nostrand Reinhold, 1993.

KNOPLICH, J. Sistema músculo-esquelético: coluna vertebral. In: MENDES, R. Patologia do trabalho. Belo Horizonte: Atheneu, 1995, p. 213-227.

LAUREL, A. C.; NORIEGA, M. Processo de produção e saúde: trabalho e desgaste operário. São Paulo: Hucitec, 1989.

LEBKOWSKI, W. J. Ageing and degeneration of human lumbar intervertebral discs. Polski Merkuriusz Lekarski, Poland, v. 13, n. 75, p. 234-237, Sept. 2002.

LEMOS, C.; RISO, P. Ossos e articulações. In: BRASILEIRO FILHO, G. et al. Patologia de Bogliolo. 3. ed. Rio de janeiro: Guanabara Koogan, 1981, p. 55-69.

LIUKE, M. et al. Disc degeneration of the lumbar spine in relation to overweight. International Journal of Obesity, London, v. 29, n. 8, p. 903-908, Aug. 2005.

LOTZ, J. C. et al. Compression-induced degeneration of the intervertebral disc: an in vivo mouse model and finite-element study. Spine, United States, v. 23, n. 23, p. 2493-2506, Dec. 1998.

MATSUMOTO, T. et al. Cyclic mechanical stretch stress increases three growth rate and collagen synthesis of nucleus pulposus cells in vitro. Spine, United States, v. 24, n. 4, p. 315-319, Feb. 1999.

MAZZONI, C. F.; COUTO, H. A. Aspectos físicos e radiológicos da coluna lombossacra de trabalhadores envolvidos em atividades pesadas comparados com indivíduos de atividades sedentárias. In: CONGRESSO DA ASSOCIAÇÃO NACIONAL DE MEDICINA DO TRABALHO, 5., 1987, Florianópolis. Anais... Florianópolis: ANAMT, 1987. p. 759-775.

McCARTHY, E.; FRASSICA, F. Pathology of bone and joint disorders: with clinical and radiographic correlation. Philadelfia: W. B. Saunders Company, 1998.

MENDES, R. Patologia do trabalho. São Paulo: Atheneu, 1995.
NACHEMSON, A.; ELFSTRON, G. Intravital dynamic pressure measurements in lumbar discs. A study of common moviments, maneuvers and exercises. Scandinavian Journal of Rehabilitation Medicine, Sweden, v.1 p. 1-40, 1970. Supplement.

NUNES, C. V. Lombalgia e lombociatalgia: diagnóstico e tratamento, Rio de janeiro: Medsi, 1989.

PINCUS, T. Clinical evidence for osteoarthritis as an inflammatory disease. Current Rheumatology Reports, United States, v. 3, n. 6, p. 524-534, Dec. 2001.

RIBEIRO, I. B. Acidente do trabalho. São Paulo: Andrei, 1979.

SALTER, R. B. Distúrbios e lesões do sistema músculoesquelético. 2. ed. Rio de Janeiro: Medsi, 1985.

SEIDLER, A. et al. The role of cumulative physical load in lumbar spine disease: risk factors for lumbar osteochondrosis and spondylosis associeted with chronic complaints. Occupational and Environmental Medicine, England, v. 58, n. 11, p. 735-746, Nov. 2001.

SELL, I. Ergonomia para profissionais de saúde ocupacional. In: VIEIRA, S. I. Medicina básica do trabalho. Curitiba: Gênesis, 1994. v. 2, p. 251-323.

SIMMONS JR., E. D. et al. Familial predisposition for degenerative disc disease. A case-control study. Spine, United States, v. 21, n. 13, p. 1527-1529, July 1996.

SKROBONJA, A.; KONTOSIC, I. Bernadino Rammazzini's de morbis artificum diatriba or three hundred years from the beginning of modern occupational medice. Arhiv za Higipenu i Toksikologuju, Croatia, v. 53, n. 1, p. 31-36, Mar. 2002.

TAYYARI, F.; SMITH, J. L. Occupational ergonomics: principles an applications. New York: Chapman \& Hall, 1997.

VIEDMAN, T.; NURMIEN, M.; TRAP, J. D. G. Lumbar spinal pathology in cadaveric material in relation to history of back pain, occupation and physical loading. Spine, United States, v. 15, n. 8, p. 728-740, Aug. 1990.

WISNER, A. A inteligencia no trabalho: textos selecionados de ergonomia. São Paulo: FUNDACENTRO, UNESP, 1994.

WORLD HEALTH ORGANIZATION. Physical status: the use and interpretation of anthropometry. Geneva: WHO, 1995. 